\title{
IL CURRICOLO FONDATO SULLE COMPETENZE ATTESE
}

\author{
Marina Diković \\ Università Juraj Dobrila di Pola \\ Facoltà di Scienze della Formazione \\ Dea Lordanić \\ Andrea Sinožić \\ Università Juraj Dobrila di Pola \\ Dipartimento di studi in lingua italiana
}

\section{Riassunto}

Gli obiettivi rappresentano l'orizzonte a breve termine e le competenze rappresentano l'orizzonte a lungo termine. Ambedue vengono definiti all'inizio del processo d'insegnamento. Le competenze attese indicano ciò che ci si attende l'alunno sarà in grado di fare una volta concluso il processo d'insegnamento. L'obiettivo della presente ricerca è quello di confrontare le competenze attese dichiarate nelle Indicazioni per il curricolo 2007 per le scuole italiane in Italia (in Gambula, 2009) e gli obiettivi formativi elencati nel Programma per l'insegnamento della lingua italiana (MSES, 2010), al fine di verificare se queste competenze attese sono collimanti con gli obiettivi formativi dichiarati nei programmi per le scuole elementari italiane in Croazia. In base all'analisi effettuata si può concludere che: 1) i descrittori degli obiettivi formativi per la scuola italiana in Italia rappresentano un possibile modello da raggiungere per gli alunni che vengono istruiti in lingua italiana nelle scuole nella Regione Istriana; 2) il curricolo fondato sulle competenze attese potrebbe essere un modello utile per valutare le conoscenze, le capacità e le abilità degli alunni e per determinare, in tal modo, le strategie che possano in futuro facilitare l'apprendimento.

Parole chiave: competenze, competenze attese, curricolo, obiettivi formativi, valutazione 


\section{Introduzione}

La scuola si assume la guida dell'alunno nel processo di acquisizione del sapere, delle abilità, delle abitudini e della formazione generale. Essa fornisce agli alunni vari strumenti e percorsi per aiutarli a raggiungere gli obiettivi didattici ${ }^{[1]}$. La considerazione dei principali elementi, temi e problemi che hanno animato (ed animano) la ricerca nel campo dell'insegnamento è stata (ed è tuttora) un tema significativo del discorso sul sistema dell'istruzione. Secondo alcuni autori (Strommillo, 2014) lo scopo degli insegnanti dovrebbe essere quello di custodire e difendere le capacità degli alunni, spesso umiliati dall'offerta istruttiva, nonché di offrire ai futuri insegnanti uno strumento mediante il quale essi possano sempre più "scoprirsi" professionisti riflessivi, volti a curare un'autentica vocazione intellettuale.

L'insegnante si confronta ogni giorno con il suo gruppo di alunni, ognuno con le sue caratteristiche peculiari, cercando di concentrarsi sulla realtà che ha di fronte. Per questo il ruolo dell'insegnante è in continuo ed incessante divenire. Il suo fine è affidato all'azione istruttiva, cioè preparare l'individuo, ovvero l'alunno, portandolo a conseguire gli obiettivi indicati nel Piano e nel programma elaborati dal Ministero. Gli insegnanti sono responsabili delle competenze attese negli alunni che sono loro affidati e devono sostenerli nei loro comportamenti emotivi, sociali, creativi ed artistici, ma soprattutto cognitivi. Devono altresì prendere in considerazione lo sviluppo individuale degli alunni e promuovere le loro capacità ed esigenze.

Il CIDREE (Consortium of Institutions for Development and Research in Education in Europe $)^{[2]}$ ha promosso una serie di confronti e di documentazioni sul rapporto fra scuola e valori. Quando educhiamo o insegniamo, trasmettiamo valori. Lesistenza stessa di una scuola è unaffermazione di valore, che di per sé mostra quello che una società deve assicurare ai giovani.

\section{Obiettivi formativi}

Il progetto di classificazione degli obiettivi formativi, delineato da Benjamin Samuel Bloom e corrispondente ai processi definiti da Anderson et al. (2001) (revisione della Tassonomia di Bloom), prevede la suddivisione degli obiettivi in tre grandi ambiti educativi: cognitivo, affettivo e psicomotorio. La distribuzione dei livelli degli obiettivi

[1] La Didattica d'insegnamento conosce tre obiettivi: obiettivo formativo, obiettivo educativo e obiettivo funzionale. L'obiettivo formativo (istruttivo) è rivolto ad imparare i concetti chiave, acquisire il sapere. Nel presente saggio è stato elaborato lobiettivo formativo che si riferisce all'istruzione, cioè al livello cognitivo.

[2] CIDREE: http://www.cidree.org/ (15 febbraio 2015) 
formativi all'interno di ciascun settore è ordinata in base ad un modello gerarchico: ogni categoria, così come le sue sottoclassi, implica un comportamento più complesso ed astratto della precedente. La tassonomia si configura come un sistema di classificazione costruito sulla base di principi pedagogici, logici e psicologici. Lordine di questi criteri non è casuale. Quanto tempo sia necessario per raggiungere un determinato livello dipende da diversi fattori, in parte anche individuali: dall'impegno, dal tempo investito e dallo sfruttamento di ulteriori offerte di esercizio. La tassonomia degli obiettivi formativi classifica tutti gli obiettivi conseguiti nell'insegnamento per far raggiungere agli alunni il livello più alto.

Il criterio psicologico ha la funzione di tener conto dei principi elementari della psicologia dell'apprendimento. Il primo, quello cognitivo, è stato costruito, studiato e applicato con maggiore ampiezza, perché le istituzioni scolastiche si orientano soprattutto verso apprendimenti cognitivi. Il settore cognitivo è stato articolato in sei classi principali, ciascuna delle quali si ramifica in sei sottoclassi: 1) Conoscenza - capacità di rievocare il materiale memorizzato, significa ricordare, riconoscere e riprodurre nella forma originale; 2) Comprensione - la facoltà di afferrare il senso di un'informazione e di saperlo trasformare; 3) Applicazione - utilizzare il materiale già conosciuto per la soluzione di nuovi problemi; 4) Analisi - separare gli elementi costitutivi della comunicazione con l'intento di osservarne i rapporti; 5) Valutazione - formulazione di giudizi sul valore del materiale e/o dei metodi in base a criteri esplicitati; 6) Sintesi - riunire gli elementi al fine di formare una nuova struttura organizzata e coerente (Anderson et al., 2001).

Gli obiettivi didattici devono accentuare in primo luogo la necessità di rilevare le capacità particolari che l'alunno dovrà aver acquisito alla fine di un corso o al termine di un processo di apprendimento particolare, quelle condotte specifiche e quelle attività osservabili che testimoniano il processo effettivo di tali capacità. Gli obiettivi (ing. goals) si pongono in una prospettiva diversa rispetto alle finalità. Mentre le finalità si riferiscono ad un proponimento ideale di tipo pedagogico, gli obiettivi si definiscono in termini più operativi e indicano i risultati che si intendono far conseguire agli alunni al termine del processo istruttivo annuale o pluriennale (Bonaiuti et al., 2008). Gli obiettivi orientano sia la natura che il significato degli interventi istruttivi predisposti, nel complesso, dagli insegnanti ai fini del più completo sviluppo possibile delle capacità di ciascun alunno. 


\section{Competenze}

Quando si dice che una persona è competente si intende valorizzare quello che una persona sa fare, indipendentemente da come ha imparato. Competenza significa dunque la capacità di svolgere bene un determinato compito o professione. Il termine competenza (usato sempre al singolare) indica quella generica qualità posseduta dalla persona che si dimostra competente (Piršl, 2014). Nel campo delle scienze sociali, la competenza viene definita come esperienza, conoscenza, desiderio e motivazione, tenendo conto dell'importanza del contesto socio-economico e culturale delle competenze basilari (Viteriti, 2005, in Piršl, 2014).

Pellerey (2007) spiega il significato della competenza descrivendola come capacità di attivare e coordinare le proprie risorse interne (conoscenze, abilità e disposizioni interne stabili) e quelle esterne disponibili per portare a termine validamente ed efficacemente i compiti richiesti o le sfide da affrontare. Sul piano pratico la competenza significa affrontare e saper inquadrare correttamente situazioni problematiche, mettere in moto le risorse richieste, gestire se stessi in maniera adeguata per conseguire il risultato atteso, controllare e valutare bene sia il risultato ottenuto, sia come lo si è ottenuto. Lo scenario delineato dalla 'società della conoscenza' (Commissione delle Comunità Europee, 2000) è caratterizzato da alcuni elementi fondamentali che ruotano intorno all'esigenza di spostare il focus dell'azione didattica dall'insegnamento all'apprendimento, dal programma al curricolo, dalle nozioni alle competenze.

Otto sono le competenze secondo il Quadro europeo delle competenze chiave (Commission of the European Communities, 2006) ${ }^{[3]}$. Sviluppare una o più competenze comporta mettere l'alunno al centro di un processo, al termine del quale si presume, sul piano teorico, che egli possa giungere a possedere in maniera significativa, stabile e fruibile concetti e quadri concettuali; abbia raggiunto adeguate abilità intellettuali e pratiche sapendo come, quando e perché valorizzarle; disponga di adeguati significati, valori, motivazioni, atteggiamenti, concezioni di sé e forme di rapporto con il sapere da acquisire, capacità di concentrazione e di perseveranza nel portare a termine gli impegni, ecc. Ad esempio: la comunicazione nella madrelingua è la capacità di manifestare e decodificare concetti, idee, sentimenti, avvenimenti sia in forma scritta che orale e di interagire in modo adeguato e creativo sul piano linguistico in ambito culturale e sociale.

[3] Comunicazione nella madrelingua, comunicazione nelle lingue straniere, competenza matematica e competenze di base in scienza e tecnologia, competenza digitale, imparare a imparare, competenze sociali e civiche, spirito di iniziativa e imprenditorialità, consapevolezza ed espressione culturale. Quadro europeo delle competenze chiave (Commission of the European Communities, 2006) - reperibile sul sito: http://ec.europa.eu/dgs/education_culture/publ/pdf/ll-learning/keycomp_it.pdf (10 febbraio 2015) 


\section{Competenze attese}

Le competenze devono essere realizzate nelle competenze attese. Il concetto di competenze attese (ingl. outcomes, cro. ishodi učenja) è inteso come competenza in un'attività e/o ambito nel quale una persona possiede il sapere, l'esperienza, la familiarità e la professionalità, oppure come competenza in unarea, attività o mestiere che coinvolge adeguate conoscenze, capacità, abilità, atteggiamenti e pratiche (Spajić-Vrkaš et al., 2001). La competenza attesa è il risultato del processo d'insegnamento ed indica la conoscenza, la comprensione e le abilità che gli alunni dovrebbero dimostrare dopo aver seguito il corso, cioè quello che si ritiene debbano essere in grado di fare al termine del processo d'insegnamento. Si tratta di "abilità e capacità, mediante il rapporto tra il che cosa deve essere appreso e il modo con cui deve avvenire" (Guasti, 2012, p. 117). Non si dovrebbe definire la competenza attesa senza raggiungere gli standard i quali rappresentano "un insieme di livelli di apprendimento descritti mediante una relazione tra conoscenza e operazione mentale" (Guasti, 2012, p. 116).

Gli obiettivi specifici (ing. objectives) risultano essere più strutturati rispetto agli obiettivi generali e spesso vengono identificati come obiettivi comportamentali (detti anche obiettivi operativi). Secondo la definizione di alcuni autori (Spajić-Vrkaš et al., 2001) gli obiettivi rappresentano la condizione da raggiungere. Il comportamento umano ha lo scopo di raggiungere gli obiettivi specifici e comportamentali. Con questi obiettivi l'insegnante precisa in modo concreto ed univoco ciò che secondo le sue previsioni l'alunno saprà fare al termine dell'apprendimento. Il processo d'insegnamento dovrebbe essere focalizzato sul raggiungimento delle competenze attese negli alunni (Matijević, Radovanović, 2011).

\section{Il concetto di curricolo (curriculum)}

Il problema del curricolo e la sua natura è stato dibattuto da vari autori (Smith, Ewing, 2002; Previšić, 2007). Marsh (1994) si avvicina al concetto di curriculum spiegandone alcune sfaccettature: l'insegnamento dal punto di vista degli alunni, degli insegnanti e dei presidi, l'insegnamento dal punto di vista della pianificazione e dello sviluppo del curriculum, nonché delle ideologie del curriculum. Jurić (2007) spiega che il curriculum coinvolge la pianificazione e la programmazione, la struttura e la valutazione del processo d'insegnamento e la realizzazione degli obiettivi didattici, gli elementi del contenuto, il controllo del raggiungimento della pianificazione e programmazione globale, i presupposti per un'efficace realizzazione del processo d'insegnamento. 
Il termine curricolo (o curriculum), tradizionalmente inteso come itinerario di formazione individuale, assume il significato di un modello operativo che serve a descrivere le varie componenti di un procedimento didattico, introducendo elementi di razionalità e di certezza (categorie, criteri organizzativi e metodologie) nell'esperienza quotidiana. Lo scopo del curricolo è recuperare il valore formativo dell'istruzione. Riprendendo una pertinente definizione di Stenhouse (1977), il curricolo è un tentativo per comunicare i principi e le connotazioni essenziali di una proposta istruttiva in una forma tale da renderla aperta all'analisi critica. Dunque, una programmazione curricolare intende collocarsi a pieno titolo nellambito della conduzione operativa di processi aperti, controllabili e comunicabili.

Esistono vari tipi di curricolo (o curriculum). Il curricolo implicito - definito da taluni autori (Seddon, 1983) curricolo nascosto (ingl. hidden curriculum) - è riferito all'apprendimento degli atteggiamenti, delle credenze, dei valori, delle ipotesi, di tutto ciò che potrebbe essere espresso in termini di regole e rituali (lorganizzazione scolastica e il contesto socio-educativo l'integrazione tra scuola, famiglia ed altre realtà territoriali). Il curricolo esplicito coinvolge invece i contenuti del Piano e del programma per l'insegnamento, gli obiettivi i metodi e le strategie didattiche, la valutazione. Gli insegnanti sono direttamente responsabili della realizzazione curricolare esplicita. Il curricolo chiuso (Paparella, 2012, pp. 189-191) comprende il contenuto del Piano e del programma già determinato senza alcuna possibilità di modificarlo, per cui l'insegnante non ha la possibilità di individualizzare il processo d'insegnamento. Per fare in modo che il processo d'insegnamento sia flessibile, l'insegnante potrebbe scegliere contenuti, metodi e forme di lavoro a livello interdisciplinare, gli alunni potrebbero essere produttivamente impegnati nelle attività d'apprendimento e d'insegnamento, la scuola potrebbe provvedere alla comunicazione insegnante-alunno-genitori. In questo caso parliamo di curricolo aperto (Paparella, 2012, pp. 189-191).

Una corretta descrizione di un modello curricolare non può prescindere dall'analisi della situazione di partenza, dalle condizioni socio-culturali (ambientali e familiari), dalla cultura dell'insegnante e degli alunni, come pure dalle loro famiglie, dallorganizzazione della scuola e dalle relative regole implicite di comportamento, dalle motivazioni generali allo studio, dai livelli di abilità e dalle conoscenze degli alunni. Deve essere presente l'individuazione degli obiettivi didattici, intendendo per obiettivi le modificazioni desiderabili nel modo di agire (obiettivi educativi), di pensare ed ideare, di creare (obiettivi funzionali) e di sapere (obiettivi formativi), come pure delle competenze. Il modello curricolare coinvolge anche la scelta dei contenuti, cioè delle materie di studio che appaiono funzionali agli obiettivi, la scelta delle procedure didattiche e dei metodi di 
insegnamento, che comprendono pure le modalità della comunicazione, l'organizzazione della classe e dei ritmi di lavoro, i materiali di lavoro, i sussidi e mezzi didattici, la messa a punto del sistema di valutazione adeguato all'oggetto sotto controllo, come pure l'analisi dei rapporti con i livelli di scolarità precedente e successiva e con le esperienze formative extracurricolari ed extrascolastiche.

Secondo Gambula (2009, pp. 15-16) esistono tre modelli di curricolo: quello incentrato sugli obiettivi (rappresenta l'elaborazione razionale di un traguardo di apprendimento), quello incentrato sui contenuti e sulle procedure (rappresenta la difficoltà di valutare i risultati) ed infine quello strutturato sulla ricerca (rappresenta la decisione intenzionale del percorso d'insegnamento). Il costruttivismo assume un approccio di carattere pragmatico, avendo focalizzato l'attenzione sul processo di costruzione dei significati e della loro comunicazione (Von Foerster, Von Glasersfeld, 2001). La costruzione del curricolo strutturato sulla ricerca presenta agli alunni la possibilità di arrivare al costruttivismo.

\section{Valutare le competenze attese}

Finita l'età dell'enciclopedismo non serve una 'scuola-spugna', ma una scuola che sappia mettere ordine, dare senso alle cose, che faccia ragionare gli alunni su quello che sanno, che sia meno ansiosa del puro apprendimento dei contenuti, e che si focalizzi su conoscenze e problemi essenziali, basati sui generatori di competenze. Ci sarà bisogno di una scuola che si fonda, non solo sull'epistemologia, ma anche sull'ermeneutica, che si propone di dare agli alunni la capacità di interpretare e trattare gli argomenti con la propria testa (Gambula, 2009). "Il focus di acquisizione delle conoscenze in un curriculum basato sulle competenze (attese) è lo sviluppo di una serie di competenze, spesso definito come competenze per la vita o competenze trasferibili piuttosto che contenuti disciplinari" (Downey et al., 2013, p. 327). Possiamo dire che ci serve una scuola che aiuti gli alunni a 'trovare il sapere' o a conquistare conoscenze che serviranno loro nella vita quotidiana anziché fornir loro conoscenze "già conosciute".

Il sapere che un alunno acquisisce durante il periodo scolastico è molto soggettivo. Certi alunni potranno imparare molte cose anche nel corso della scuola elementare, mentre altri dovranno arrivare alla scuola media superiore per avere un sapere ampio e saper fare tutto senza l'aiuto di altri. Il curriculum fondato sulle competenze attese significa che non si valuta solo ciò che l'alunno sa fare, ma anche come sa farlo, cioè verrà preso in considerazione anche il percorso fatto per raggiungere i risultati. Ciò significa 
che, rispetto al punto di partenza, verranno valutati i progressi ed i miglioramenti dei singoli alunni. Tutto questo vuol dire che nell'insegnamento si deve porre attenzione al percorso di miglioramento dell'alunno per giungere ad un'acquisizione del sapere consapevole e responsabile.

Un curricolo innovativo dovrebbe ridefinire la distinzione tra valutazione dell'apprendimento e valutazione per l'apprendimento. La valutazione dell'apprendimento registra il livello di competenza raggiunto, propone prove d'ingresso per una collocazione iniziale o valutazioni a ciclo breve o esami conclusivi, documenta la padronanza degli standard per decidere il passaggio alla classe successiva. Di fronte a questo tipo di valutazione l'alunno studia per raggiungere gli standard e i risultati più alti, svolge prove il cui superamento costituisce il fine dello studio. La valutazione per lapprendimento mette invece gli alunni in condizione di comprendere lo stadio di sviluppo nel quale collocare il proprio livello di padronanza, aiuta gli alunni a conseguire tutti gli obiettivi già previsti, sostiene e promuove il miglioramento continuo verso il successo. Nel caso invece in cui i risultati di apprendimento ed i comportamenti degli alunni non siano quelli ipotizzati nella programmazione, è eticamente corretto convenire che non sono "sbagliati" gli alunni, bensì è l'attività progettuale predisposta a contenere in sé sanabili o insanabili anomalie, a seconda della cospicuità dell'insuccesso registrato (Ghelfi, Lelli, 2000).

La valutazione fondata sui feedback descrittivi degli alunni prevede la costruzione di strumenti concordati e condivisi tra insegnanti e alunni per la definizione delle caratteristiche dei prodotti attesi ${ }^{[4]}$, per il sostegno all'autovalutazione e per lo sviluppo delle competenze metacognitive, ovvero il livello più alto del settore cognitivo (Tassonomia di Bloom). Di fronte alla valutazione per l'apprendimento, l'alunno è coinvolto nella costruzione di un sistema di auto-/etero-valutazione ed opera sui risultati di valutazione della classe per migliorare le proprie prestazioni. Partendo dagli aspetti semantici, dall'oggetto di studio e dalle finalità, l'analisi dell'insegnamento potrebbe entrare nel vivo dei diversi approcci e modelli di valutazione, per occuparsi infine degli strumenti e dei metodi docimologici per la valutazione dei risultati, indicatore dell'efficacia dell'attività di insegnamento (Schiedi, 2012). Creare una valutazione in base al curricolo fondato sulle competenze attese significa raggiungere i livelli più alti della Tassonomia di Bloom per poter valutare le conoscenze, le capacità e le abilità degli alunni.

[4] Nell'insegnamento si usano alcuni strumenti di controllo: la registrazione (check list), gli schemi di categorie osservazionali, le griglie di correzione, le rubriche, ecc. La valutazione formativa (la valutazione durante l'insegnamento) dovrebbe mirare a monitorare il processo d'apprendimento per poi apportare gli opportuni correttivi all'insegnamento/apprendimento. 


\section{Metodologia della ricerca}

\section{Finalità e ipotesi}

La finalità primaria della presente ricerca è il confronto tra le competenze attese $^{[5]}$ del documento Indicazioni per il curricolo 2007 ${ }^{[6]}$ (di seguito IC 2007) per le scuole italiane in Italia (in Gambula, 2009) e gli obiettivi formativi del Programma per l'insegnamento della lingua italiana (MSES, 2010) (di seguito PLI 2010). L'ipotesi da cui si parte è la possibilità che tali quadri teorici possano essere correlati con gli obiettivi formativi nei programmi per le scuole elementari italiane in Croazia. In secondo luogo, verrà analizzata l'eventualità che il curricolo fondato sulle competenze attese possa essere un modello utile per valutare le conoscenze, le capacità e le abilità degli alunni.

\section{Scopi specifici}

Gli scopi specifici della presente ricerca sono stati i seguenti:

1) individuare nelle competenze attese gli obiettivi formativi dei campi d'insegnamento: espressione orale e scritta, comprensione della lingua orale e scritta (ascolto e lettura) e produzione orale e scritta (parlato e scritto) del PLI 2010 in base alla Tassonomia di Bloom (Anderson et al., 2001)

2) valutare l'acquisizione delle competenze attese da parte degli alunni nei suddetti campi d'insegnamento.

\section{Partecipanti e strumento}

Per raggiungere gli scopi specifici sono stati intervistati gli insegnanti della quarta classe e gli insegnanti di lingua italiana dell'ottava classe delle scuole elementari ${ }^{[7]}$ della Regione Istriana (Repubblica di Croazia).

Lo strumento di analisi del Programma per l'insegnamento della lingua italiana è stata la matrice che ha compreso i descrittori delle competenze attese che fanno parte delle IC 2007 (in Gambula, 2009) per le scuole italiane in Italia, gli obiettivi formativi dei tre campi d'insegnamento del PLI 2010 e i sei livelli della Tassonomia di Bloom. La

\footnotetext{
[5] La descrizione degli obiettivi formativi della disciplina (Gambula, 2009, p. 127), in questo lavoro viene considerata come la descrizione delle competenze attese (ingl. outcomes; cro. ishodi učenja).

[6] Il processo educativo nelle scuole italiane, dall'anno 2012/2013, si svolge in conformità alle Indicazioni nazionali per il curricolo 2012 (INC 2012) reperibili sul sito http://www.indicazioninazionali.it. Il motivo per cui, nella presente ricerca, abbiamo preso in considerazione l' IC 2007, sta nel fatto che Gambula (2009) tratta l'argomento 'curricolo' basandosi sul documento IC 2007. Confrontando le competenze attese indicate nell' IC 2007 e nell' INC 2012 possiamo notare che queste sono simili nei due documenti.

[7] Il sistema educativo-istruttivo, in Croazia, comprende otto classi di scuola elementare, suddivise in quattro inferiori e quattro superiori.
} 
valutazione dell'acquisizione delle competenze attese da parte degli alunni, sia per le classi inferiori che per quelle superiori, si è basata sull'analisi delle prove scritte e orali in lingua italiana secondo i tre campi d'insegnamento.

\section{Procedimento}

Le competenze attese nel documento IC 2007 sono suddivise in quattro ambiti: percepire, catalogare, formulare ipotesi, risolvere problemi.

I campi d'insegnamento del PLI 2010 presi in esame in quest'analisi sono: a) espressione orale e scritta, b) comprensione della lingua orale e scritta (ascolto e lettura), c) produzione orale e scritta (parlato e scritto) ${ }^{[8]}$. Il primo campo è stato esaminato per la quarta classe e gli ultimi due per lottava. Il motivo della scelta dei campi qui sopra indicati sta nel fatto che i contenuti (le competenze attese) dal documento IC 2007 corrispondono, per l'appunto, a tali campi. Inoltre, sono state prese in considerazione la quarta e l'ottava classe perché le competenze attese del documento IC 2007 corrispondono, di caso in caso, sia alle classi inferiori che alle classi superiori della scuola elementare.

I quattro ambiti delle IC 2007 e le risposte degli insegnanti intervistati sono stati paragonati, al termine della ricerca, con i sei livelli della tassonomia di Bloom, dato che essa rappresenta uno dei modi per formalizzare le fasi di acquisizione e familiarizzazione con i set di informazioni o teorie e viene utilizzata in psicologia dell'educazione per definire le fasi dell'apprendimento e costruire il processo educativo (Anderson et al., 2001).

Agli insegnanti è stato spiegato come procedere e cosa dovevano analizzare nella matrice d'analisi appositamente costruita per questa ricerca, nonché come valutare l'acquisizione delle competenze degli alunni nella lingua italiana (limitatamente ai campi d'insegnamento indicati) nelle prove scritte e orali. Un limite della ricerca potrebbe consistere nel fatto che non si è presa in considerazione la differenza tra gli alunni per i

\footnotetext{
[8] Nel Programma per l'insegnamento della lingua italiana (MSES, 2010) per ogni tema vengono descritti gli obiettivi formativi. Ad esempio, per il campo espressione orale e scritta (per la quarta classe): consolidare il racconto orale esteso e conciso; distinguere il fatto principale da quelli secondari; conversare su un argomento; conoscere gli elementi della comunicazione verbale; individuare e riconoscere gli elementi della comunicazione gestuale; scrivere correttamente, mettendo in pratica tutte le nozioni apprese; usare correttamente la maiuscola e le difficoltà ortografiche, saper usare le abbreviazioni; ecc. Per il campo comprensione della lingua orale e scritta (ascolto e lettura) (per lottava classe): cercare informazioni particolari all'interno di un testo; applicare diverse strategie di lettura secondo i tipi di testo (narrativo, descrittivo, espositivo, regolativo, argomentativo), ovvero leggere in modo espressivo, leggere per studiare; migliorare la velocità di lettura; muovere rapidamente e correttamente gli occhi; ampliare il proprio arco visivo; esercitare la velocità di lettura e la capacità di concentrazione; ecc. e per il campo produzione orale e scritta (parlato e scritto) (sempre per lottava classe elementare): produrre testi diversi partendo da un unico testo-base; riscrivere testi letterari con procedure creative guidate, applicando manipolazioni a livello stilistico (cambiare il punto di vista, il registro linguistico...); elaborare testi con programmi di videoscrittura; riconoscere e riprodurre testi in prosa: diari, lettere, racconti, relazioni...); scrivere in modo creativo, riconoscere e utilizzare alcuni semplici artifici retorici; scrivere il commento di un testo narrativo; ecc.
} 
quali la lingua italiana è lingua materna e quelli per i quali la lingua italiana non è, invece, lingua materna, bensì soltanto lingua oggetto d'insegnamento.

\section{Discussione}

Nell'analisi dei descrittori delle competenze attese che fanno parte delle IC 2007 (in Gambula, 2009) per le scuole italiane in Italia, degli obiettivi formativi dei tre campi d'insegnamento del PLI 2010 e dei livelli della Tassonomia di Bloom in base alle matrici, gli insegnanti della quarta classe elementare hanno evidenziato che, nell'ambito della percezione, non tutti gli alunni riescono ad intuire il significato globale del testo, perché prestano maggior attenzione alla tecnica di lettura piuttosto che al contenuto del testo stesso. Linsegnamento si basa sull'esposizione orale e sulle tecniche di lettura. La maggior parte degli alunni riesce a capire anche il contenuto di un testo, ma gli alunni con minor competenza linguistica in lingua italiana hanno difficoltà di apprendimento. Gli alunni riescono a capire meglio il contenuto ed il significato globale di un testo ascoltandolo, piuttosto che leggendolo, in quanto la difficoltà della lettura li induce a concentrarsi sulle singole parole che vanno leggendo, col conseguente effetto di perdere di vista il livello del contenuto.

Nell'ambito del catalogare, gli alunni sono in grado di classificare gli elementi di un testo secondo criteri logici, spaziali e lessicali. Gli alunni della quarta classe inferiore hanno molta difficoltà con gli elementi morfologici e sintattici, probabilmente perché non sono di madre lingua italiana. Alcuni alunni trovano difficoltà nell'uso dei digrammi: $c h$, $g h$, ce e gn, e fanno errori ortografici, soprattutto confondendo $e$ con $\dot{e}, o$ con ho ed $a$ con ha. Commettono maggiori errori coloro che hanno una minore competenza linguistica per l'italiano, oppure quelli che hanno la padronanza di un dialetto, istroveneto o istrioto (o/ho - go, $a / h a-g a)$. Soprattutto, gli alunni non distinguono correttamente la congiunzione $e$ dalla $3^{\text {a }}$ pers. sing. del Presente Indicativo del verbo essere $(e / \grave{e})$.

Considerando l'ambito della formulazione di ipotesi, appena in quarta classe gli alunni riconoscono la tipologia di un testo: se si tratta di un dialogo, un monologo, una narrazione o una descrizione, sono in grado di individuarne il tema centrale e la struttura. 
Tabella 1. Confronto dei descrittori delle competenze attese nei quattro ambiti secondo le IC 2007 per le scuole italiane in Italia e delle competenze attese degli alunni della quarta classe elementare secondo le risposte degli insegnanti intervistati in base al PLI 2010 ed ai livelli della Tassonomia di Bloom

\begin{tabular}{|c|c|}
\hline $\begin{array}{l}\text { Descrittori delle competenze } \\
\text { attese (Italia) }\end{array}$ & $\begin{array}{l}\text { Competenze attese (Croazia) - risposte degli } \\
\text { insegnanti }\end{array}$ \\
\hline \multicolumn{2}{|r|}{ PERCEPIRE } \\
\hline $\begin{array}{l}\text { - riconosce il significato globale del } \\
\text { testo (ascoltato/letto) }\left(1^{\circ}\right)^{[9]} \\
\text { - in un testo individua le } \\
\text { informazioni relative agli elementi } \\
\text { della struttura base }\left(4^{\circ}\right) \\
\text { - trova spunti dagli elementi della } \\
\text { struttura base del testo per parlare } \\
\text { o scrivere }\left(3^{\circ}\right)\end{array}$ & $\begin{array}{l}\text { - capisce il contenuto di un testo }\left(2^{\circ}\right) \\
\text { - analizza la struttura del testo }\left(4^{\circ}\right) \\
\text { - riconosce i tipi di testo }\left(1^{\circ}\right) \\
\text { - individua le informazioni relative agli elementi della } \\
\text { struttura base, come pure il significato di un testo letto } \\
\left(4^{\circ}\right)\end{array}$ \\
\hline \multicolumn{2}{|r|}{ CATALOGARE } \\
\hline $\begin{array}{l}\text { - classifica gli elementi di un } \\
\text { testo secondo criteri dati (logici, } \\
\text { spaziali, lessicali, morfologici, } \\
\text { sintattici) }\left(4^{\circ}\right) \\
\text { - riconosce le caratteristiche } \\
\text { essenziali delle diverse tipologie } \\
\text { testuali }\left(3^{\circ}\right)\end{array}$ & $\begin{array}{l}\text { - classifica gli elementi di un testo secondo criteri logici, } \\
\text { spaziali e lessicali }\left(4^{\circ}\right)\end{array}$ \\
\hline \multicolumn{2}{|c|}{ FORMULARE IPOTESI } \\
\hline $\begin{array}{l}\text { - dato un semplice testo, formula } \\
\text { ipotesi sull'appartenenza al genere } \\
\left(6^{\circ}\right) \\
\text { - dato un testo, formula ipotesi } \\
\text { sul contenuto, su termini ed } \\
\text { espressioni avvalendosi del } \\
\text { contesto delle immagini, della } \\
\text { titolazione }\left(6^{\circ}\right) \\
\text { - ipotizza variabili diverse in uno } \\
\text { stesso testo }\left(6^{\circ}\right)\end{array}$ & $\begin{array}{l}\text { - riconosce la strofa, versi ed anche la cronologia dei } \\
\text { fatti ed i protagonisti, antagonisti }\left(1^{\circ}\right) \\
\text { - risponde alle domande come il luogo dell'avvenimento, } \\
\text { il tempo, le vicende, i protagonisti, i personaggi } \\
\text { secondari, l'antagonista }\left(2^{\circ}\right) \\
\text { - riconosce il tema e i tipi di poesia: filastrocche, } \\
\text { canzoni, barzellette, riconoscono anche la rima: } \\
\text { baciata, incrociata }\left(1^{\circ}\right) \\
\text { - distingue il racconto reale da quello fantastico }\left(2^{\circ}\right) \\
\text { - riconosce la tipologia di un testo (dialogo, monologo, } \\
\text { narrazione, descrizione) }\left(1^{\circ}\right) \\
\text { - individua il tema centrale e la struttura del testo }\left(1^{\circ}\right)\end{array}$ \\
\hline \multicolumn{2}{|r|}{ RISOLVERE PROBLEMI } \\
\hline $\begin{array}{l}\text { - rielabora la struttura di un testo } \\
\text { (riscrittura, parafrasi, riassunto) } \\
\left(6^{\circ}\right) \\
\text { - organizza un breve discorso orale } \\
\text { adeguato agli scopi e ai destinatari } \\
\left(6^{\circ}\right) \\
\text { - produce testi scritti adeguati agli } \\
\text { scopi e ai destinatari (descrizioni, } \\
\text { racconti, lettere, resoconti, diari) } \\
\left(6^{\circ}\right) \\
\text { - produce testi corretti dal punto di } \\
\text { vista ortografico, morfosintattico e } \\
\text { lessicale }\left(6^{\circ}\right)\end{array}$ & $\begin{array}{l}\text { - scrive brevi temi liberi }\left(6^{\circ}\right) \\
\text { - racconta il riassunto di un testo/ letture domestiche } \\
\left(3^{\circ}\right) \\
\text { - racconta un parere personale del libro }\left(2^{\circ}\right) \\
\text { - drammatizza il testo dato }\left(6^{\circ}\right) \\
\text { - ricorda le battute }\left(2^{\circ}\right) \\
\text { - capisce il testo }\left(2^{\circ}\right) \\
\text { - organizza dibattiti con i compagni di classe }\left(6^{\circ}\right) \\
\text { - rielabora testi manipolandoli, parafrasandoli, } \\
\text { completandoli, trasformandoli }\left(6^{\circ}\right)\end{array}$ \\
\hline
\end{tabular}


Per quanto riguarda l'ambito della risoluzione di problemi, gli alunni fanno molti errori grammaticali e ortografici. Fanno esposizioni orali: espongono il riassunto di un testo, leggono le letture domestiche (vengono elaborate solamente le tracce del testo, senza il riassunto, che gli alunni raccontano durante l'ora di lezione con l'aiuto dell'insegnante). È molto importante dar loro delle illustrazioni da descrivere; nonostante ciò, tuttavia, le descrizioni, se non sono guidate dall'insegnante, tendono ad essere molto superficiali. Avendo una buona padronanza del metodo uditivo, comunque, gli alunni capiscono i concetti, leggendoli, per cui sarebbe opportuno che i testi venissero più ascoltati che letti.

Confrontando i descrittori delle competenze attese nei quattro ambiti secondo le IC 2007 per le classi inferiori delle scuole italiane in Italia e le competenze attese secondo le risposte degli insegnanti intervistati in base al PLI 2010 ed ai livelli della Tassonomia di Bloom (Tabella 1) si può dedurre che le competenze attese in Italia vengono classificate con dei livelli formativi più alti di quelle attese in Croazia. Come si può osservare nella tabella 1 i livelli formativi sono più alti negli ambiti formulare ipotesi e risolvere problemi. Nessuna risposta degli insegnanti non ha superato i livelli del documento IC 2007.

Gli insegnanti dell'ottava classe, per quanto riguarda l'ambito della percezione, hanno risposto che gli alunni svolgono degli esercizi con l'aiuto dell'insegnante o dei genitori e che non riescono ad arrivare al quinto ed al sesto livello. Per catalogare, solo certi alunni trovano le analogie e le differenze tra testi diversi, ma molti hanno bisogno di qualcuno che li aiuti a confrontare i testi ed individuare questi elementi.

Per i quattordicenni è ancora molto difficile formulare delle ipotesi, probabilmente perché, nelle classi inferiori, le uniche persone che formulano ipotesi sono i loro insegnanti. Gli alunni non sono in grado di risolvere gli esercizi da soli e chiedono aiuto. E solo nelle classi superiori che si impara a formulare ipotesi, ma gli alunni non sono ancora in grado di farlo con successo. Solo coloro che hanno una maggiore competenza linguistica in italiano sono in grado di risolvere problemi, mentre la maggior parte dei restanti alunni non è ancora in grado di valutare i testi o discuterne. Nelle discussioni sanno dare soltanto delle opinioni personali, ma mancano della capacità di trarre conclusioni generali e di delineare sintesi problematiche.

[9] I livelli degli obiettivi formativi della Tassonomia di Bloom: $1^{\circ}$ Conoscere; $2^{\circ}$ Comprendere; $3^{\circ}$ Applicare; $4^{\circ}$ Analizzare; $5^{\circ}$ Valutare; $6^{\circ}$ Sintetizzare. 
Tabella 2. Confronto dei descrittori delle competenze attese nei quattro ambiti secondo le IC 2007 per le scuole italiane in Italia e delle competenze attese degli alunni dellottava classe elementare secondo le risposte degli insegnanti intervistati in base al PLI 2010 ed ai livelli della Tassonomia di Bloom

\begin{tabular}{|c|c|}
\hline Descrittori delle competenze attese (Italia) & $\begin{array}{l}\text { Competenze attese (Croazia) - } \\
\text { risposte degli insegnanti }\end{array}$ \\
\hline \multicolumn{2}{|l|}{ PERCEPIRE } \\
\hline $\begin{array}{l}\text { - riconosce il significato globale del testo (ascoltato, } \\
\text { letto) }\left(2^{\circ}\right) \\
\text { - in un testo individua le informazioni relative agli } \\
\text { elementi della struttura base e al punto di vista } \\
\text { dell'interlocutore }\left(4^{\circ}\right) \\
\text { - verbalizza gli elementi della struttura base del testo } \\
\left(3^{\circ}\right)\end{array}$ & $\begin{array}{l}\text { - riconosce il significato globale } \\
\text { del testo (ascoltato, letto) }\left(2^{\circ}\right) \\
\text { - verbalizza gli elementi della } \\
\text { struttura base del testo }\left(3^{\circ}\right)\end{array}$ \\
\hline \multicolumn{2}{|l|}{ CATALOGARE } \\
\hline $\begin{array}{l}\text { - classifica/correla gli elementi di un testo secondo } \\
\text { criteri dati (logici, spaziali, lessicali, morfologici, } \\
\text { sintattici) }\left(4^{\circ}\right) \\
\text { - distingue le diverse tipologie testuali }\left(2^{\circ}\right) \\
\text { - confronta testi dello stesso genere o di generi diversi/ } \\
\text { dello stesso autore o di autori diversi individuando } \\
\text { analogie e differenze }\left(5^{\circ}\right)\end{array}$ & $\begin{array}{l}\text { - riconosce le diverse tipologie dei } \\
\text { testi }\left(1^{\circ}\right) \\
\text { - classifica i testi letti in categorie } \\
\text { diverse }\left(4^{\circ}\right) \\
\text { - trova le analogie e le differenze } \\
\text { tra testi diversi }\left(3^{\circ}\right)\end{array}$ \\
\hline \multicolumn{2}{|l|}{ FORMULARE IPOTESI } \\
\hline $\begin{array}{l}\text { - dato un testo, formula ipotesi motivate } \\
\text { sull'appartenenza al genere facendo riferimento ai suoi } \\
\text { elementi strutturali }\left(6^{\circ}\right) \\
\text { - dato un testo, formula ipotesi motivate sulla funzione } \\
\text { logica e sul significato contestuale di termini, } \\
\text { espressioni, elementi }\left(6^{\circ}\right) \\
\text { - riferisce il testo all'intenzione comunicativa dell'autore } \\
\left(2^{\circ}\right) \\
\text { - formula ipotesi motivate sul rapporto tra il testo } \\
\text { e l'ambito storico, culturale e sociale in cui è stato } \\
\text { prodotto }\left(6^{\circ}\right)\end{array}$ & $\begin{array}{l}\text { - riconosce il genere facendo } \\
\text { riferimento ai suoi elementi } \\
\text { strutturali }\left(2^{\circ}\right) \\
\text { - riconosce il testo e l'ambito } \\
\text { storico, culturale e sociale in cui è } \\
\text { stato prodotto }\left(2^{\circ}\right)\end{array}$ \\
\hline \multicolumn{2}{|l|}{ RISOLVERE PROBLEMI } \\
\hline $\begin{array}{l}\text { - produce un testo scritto (riassunto, riscrittura, } \\
\text { parafrasi, appunti...) trasformando una struttura data } \\
\left(6^{\circ}\right) \\
\text { - espone oralmente in modo adeguato agli scopi e ai } \\
\text { destinatari (dialogo, esposizione pianificata...) }\left(6^{\circ}\right) \\
\text { - pianifica e produce testi scritti adeguati agli scopi } \\
\text { e ai destinatari (testi descrittivi, narrativi, espositivi, } \\
\text { argomentativi) }\left(6^{\circ}\right) \\
\text { - produce testi rispettando le convenzioni ortografiche } \\
\text { ed i criteri morfologici, sintattici e lessicali }\left(6^{\circ}\right) \\
\text { - rivede il testo durante la stesura valutandone la } \\
\text { pertinenza e operando le opportune correzioni }\left(5^{\circ}\right) \\
\text { - valuta e discute opinioni, affermazioni, tesi }\left(5^{\circ}\right)\end{array}$ & $\begin{array}{l}\text { - scrive riassunti ed appunti in } \\
\text { base al piano e in base alla lettura } \\
\left(3^{\circ}\right) \\
\text { - scrive brevi testi narrativi, } \\
\text { informativi adeguati ai diversi } \\
\text { scopi e destinatari }\left(6^{\circ}\right) \\
\text { - spiega l'opinione personale }\left(2^{\circ}\right)\end{array}$ \\
\hline
\end{tabular}


Confrontando i descrittori delle competenze attese nei quattro ambiti secondo le IC 2007 per le classi superiori delle scuole italiane in Italia e le competenze attese secondo le risposte degli insegnanti intervistati in base al PLI 2010 ed ai livelli della Tassonomia di Bloom (Tabella 2), si possono evidenziare alti livelli soltanto negli ambiti della formulazione di ipotesi e risoluzione di problemi nel documento IC 2007. A parte ciò, non è dato notare, nelle risposte degli insegnanti intervistati, livelli alti corrispondenti alla Tassonomia di Bloom.

\section{Conclusione}

In base alla presente ricerca, confrontando i descrittori delle competenze attese nei quattro ambiti secondo le Indicazioni per il curricolo 2007 per le classi inferiori delle scuole italiane in Italia e le competenze attese secondo le risposte degli insegnanti intervistati in base al Programma per l'insegnamento della lingua italiana (MSES, 2010) ed ai livelli della Tassonomia di Bloom (Anderson et al., 2001), si può dedurre che le competenze attese in Italia vengono classificate con dei livelli formativi più alti di quelli attesi in Croazia e che nessuna delle risposte degli insegnanti (Croazia) non ha superato i livelli del documento Indicazioni per il curricolo 2007. Confrontando i descrittori delle competenze attese per le classi superiori delle scuole italiane in Italia e le competenze attese secondo le risposte degli insegnanti, si possono evidenziare alti livelli negli ambiti del formulare ipotesi e risolvere problemi. A parte ciò, non è dato osservare, nelle risposte degli insegnanti intervistati in Croazia, il raggiungimento dei livelli più alti della Tassonomia di Bloom.

In base all'analisi esposta possiamo concludere che: 1) i descrittori degli obiettivi formativi per la scuola italiana in Italiana rappresentano un possibile modello da raggiungere per gli alunni delle scuole della Regione Istriana che vengono istruiti in lingua italiana; 2) il curricolo fondato sulle competenze attese potrebbe essere un modello utile per valutare le conoscenze, le capacità e le abilità degli alunni e per determinare in tal modo le strategie per facilitare l'apprendimento. Questo problema potrebbe costituire l'argomento di future ricerche nell'ambito della didattica della lingua italiana per definire i metodi d'insegnamento e le strategie utili per un apprendimento di successo. 


\section{BIBLIOGRAFIA}

ANDERSON, L. W., KRATHWOHL, D. R., AIRASIAN, P. W., CRUIKSHANK, K. A., MAYER, R. E., PINTRICH, P. R., RATHS, J., WITTROCK, M. C., 2001, A Taxonomy for Learning, Teaching, and Assessing: A Revision of Bloom's Taxonomy of Educational Objectives, Longman, New York.

BONAIUTI, G., CALVANI, A., RANIERI, M., 2008, Fondamenti di didattica, Carocci, Roma.

COMMISSION OF THE EUROPEAN COMMUNITIES, 2006, Recommendation of the European Parliament and of the Council of 18 December 2006 on key competences for lifelong learning. Brussels. Reperibile alla pagina web: http://eur-lex.europa.eu/ LexUriServ/site/en/oj/2006/1_394/1_39420061230en00100018.pdf (15 febbraio 2015)

COMMISSIONE DELLE COMUNITÀ EUROPEE, 2000, Memorandum sull'istruzione e la formazione permanente. SEC(2000) 1832, Bruxelles. Reperibile alla pagina web: http://archivio.pubblica.istruzione.it/dg_postsecondaria/memorandum.pdf (15 febbraio 2015)

DOWNEY, CH., BYRNE, J., SOUZA, A., 2013, Researching the CompetenceBased Curriculum: Preface to a Case Study of Four Urban Secondary Schools, Curriculum Journal, 24(3), 321-334.

GAMBULA, G. (ed.), 2009, Il curricolo verticale dai 3 ai 14 anni. Competenze, saperi, modelli e metodologie, Centro di Iniziativa Democratica degli Insegnanti, Milano.

GHELFI, D., LELLI, L., 2000, Manuale di didattica per la scuola elementare, Laterza, Roma-Bari.

GUASTI, L., 2012, Didattica per competenze. Orientamenti e indicazioni pratiche, Erickson, Trento.

JURIĆ, V., 2007, Kurikulum suvremene škole, in PREVIŠIĆ, V. (ed.), Kurikulum: teorije-metodologija-sadržaj-struktura, Zavod za pedagogiju, Školska knjiga, Zagreb, 253-307.

MARSH, C. J., 1994, Kurikulum, Educa, Zagreb.

MINISTERO DELLLA SCIENZA, DELL'EDUCAZIONE E DELLO SPORT DELLA REPUBBLICA DI CROAZIA, 2010, Programma per l'insegnamento della lingua italiana per le scuole elementari italiane.

PAPARELLA, N., 2012, L'agire didattico, Alfredo Guida Editore, Università Telematica Pegaso, Napoli. 
PELLEREY, M., 2007, Programmazione, in CERINI, G., SPINOSI, M. (a cura di), Voci della scuola, VI, Napoli, Tecnodid, 391-402.

PIRŠL, E., 2014, (Re)definicija pojma kompetencije i interkulturalne kompetencije, in HRVATIĆ, N. (ed.), Interkulturalno obrazovanje i europske vrijednosti, Odsjek za pedagogiju - Filozofski fakultet u Zagrebu, Visoka škola za menadžment u turizmu i informatici u Virovitici, Zagreb - Virovitica, 47-67.

PREVIŠIĆ, V. (ed.), 2007, Kurikulum: teorije-metodologija-sadržaj-struktura, Zavod za pedagogiju, Školska knjiga, Zagreb.

SCHIEDI, A., 2012, La valutazione nella scuola. Aspetti, modelli ed esperienze, Edises, Napoli.

SEDDON, T., 1983, The Hidden Curriculum: An Overview, in Curriculum Perspectives, 3, 1-6.

SMITH, D., EWING, R., 2002, Curriculum Studies, in Change: Transformations in Education, 5(I), 26-45.

SPAJIĆ-VRKAŠ, V., KUKOČ, M., BAŠIĆ, S., 2001, Obrazovanje za ljudska prava i demokraciju: Interdisciplinarni rječnik, Hrvatska komisija za UNESCO i Projekt „Obrazovanje za mir i ljudska prava za hrvatske osnovne škole“, Zagreb.

STENHOUSE, L., 1977, Dal programma al curricolo. Politica, burocrazia e professionalità, Armando, Roma.

STROMMILLO, M., 2014, L'armadio della didattica. Un percorso educativo per la libertà, Armando, Roma.

VON FOERSTER, H., VON GLASERSFELD, E., 2001, Come ci si inventa. Storie, buone ragioni ed entusiasmi di due responsabili dell'eresia costruttivista, Odradek, Roma. 


\section{KURIKULUM TEMELJEN NA ISHODIMA UČENJA}

Ciljevi predstavljaju kratkoročne mete, a kompetencije dugoročne. Oblikuju se na početku nastavnoga procesa. Ishodi učenja označavaju ono za što će učenik biti osposobljen na kraju nastavnoga procesa. Problem ovoga rada jest usporediti ishode učenja dokumenta Indicazioni per il curricolo 2007 za talijanske škole u Italiji (u Gambula, 2009) i obrazovne ciljeve Programa za nastavu talijanskoga jezika (Programma per l'insegnamento della lingua italiana, MSES, 2010) kako bi se moglo zaključiti jesu li ishodi učenja u korelaciji s obrazovnim ciljevima osnovnih škola u Republici Hrvatskoj. S obzirom na analizu možemo zaključiti: 1) deskriptori obrazovnih ciljeva za talijanske škole u Italiji predstavljaju jedan mogući model za učenike u Istarskoj županiji koji se poučavaju na talijanskome jeziku; 2) kurikulum temeljen na ishodima učenja mogao bi biti koristan model u vrednovanju znanja, sposobnosti i vještina učenika, kao i u određivanju strategija radi lakšega učenja.

Ključne riječi: kompetencije, ishodi učenja, kurikulum, obrazovni ciljevi, vrednovanje

\section{A CURRICULUM BASED ON LEARNING OUTCOMES}

Goals are to be accomplished in the short-term and competencies in the longterm. Both should inform the teaching process at the very beginning. Learning outcomes indicate what the student will be able to do at the end of the teaching process. The aim of this study is to compare the learning outcomes of the document Indicazioni per il curricolo 2007 for the Italian school in Italy (in Gambula, 2009) and the educational objectives of the Programme for teaching Italian language (MSES, 2010) in order to conclude whether learning outcomes are in correlation with the educational goals of elementary schools in the Republic of Croatia. Based on analysis, we can conclude that: 1) the descriptors of educational goals for the Italian school in Italy represent a possible model for students in the County of Istria, who are taught in Italian; 2) a curriculum based on learning outcomes could be a useful model in the evaluation of students' knowledge, abilities and skills, as well as in identifying strategies to facilitate learning.

Keywords: competencies, learning outcomes, curriculum, educational objectives, evaluation 